\title{
PARTICIPACIÓN SOCIAL, UN FACTORA CONSIDERAR EN LA EVALUACIÓN CLÍNICA DEL ADULTO MAYOR: UNA REVISIÓN NARRATIVA
}

\author{
Walter Sepúlveda-Loyola (101,2,a, Rosielma Dos Santos Lopes (10), \\ Renata Pires Tricanico Maciel (10), 1,2, , Vanessa Suziane Probst (10),2,d \\ Program of Masters and Doctoral degree in Rehabilitation Sciences, Londrina State University (UEL) and University \\ North of Parana (UNOPAR), Londrina, Brazil. \\ 2 Grupo de Estudo de Envelecimento (GEE), Londrina State University (UEL), Londrina, Brazil. \\ 3 Program of Laws, University Center Philadelphia (UNIFIL), Londrina, Brazil. \\ ${ }^{a}$ Kinesiólogo, Magister y Candidato a Doctor en Ciencias de la Rehabilitación, ${ }^{\mathrm{b}}$ Abogada, Especialista en Derecho \\ Penal, estudiante de Magister en Derecho Negocial, ${ }^{c}$ Fisioterapeuta, estudiante de Magister em Ciencias de la \\ Rehabilitación, ${ }^{\mathrm{d}}$ Fisioterapeuta, Magister y Doctor en ciencias de la Rehabilitación y Fisioterapia.
}

\section{RESUMEN}

La participación social es definida como la integración de las personas a actividades de la comunidad en grupos sociales voluntarios u obligatorios, formales e informales, lo cual podría tener consecuencias en la salud de la población adulta mayor. Fue realizada una búsqueda en Pubmed, Scielo, Scopus y Google Scholar. Para esta revisión se analizaron 16 artículos que estudiaban el impacto de la participación social en adultos mayores, incluyendo un total de 73698096 individuos de Norteamérica, Asia, Europa, y América latina. La participación social es considerada como un factor protector para la salud mental y física de los adultos mayores, la cual ha sido asociada a disminuciones de la discapacidad, comorbilidades y mortalidad. Por esta razón, se sugiere que debe ser evaluada en la práctica clínica. Esto permitiría orientar y derivar a los adultos mayores a participar de determinadas organizaciones comunitarias, principalmente aquellos que no tienen redes de apoyo, que no están vinculados a grupos comunitarios, que tienen síntomas de depresión o que están iniciando un cuadro de deterioro físico o cognitivo. De este modo, la salud pública podría aumentar sus acciones de prevención y promoción de la salud por medio de las organizaciones comunitarias. Por otro lado, la falta de instrumentos y consensos para evaluar la participación social fue discutida en esta revisión donde se ha propuesto un cuestionario de evaluación de la participación social del adulto mayor que debe ser validado y estudiado en el futuro.

Palabras claves: Participación Social; Envejecimiento; Funcionalidad; Salud. (fuente: DeCS BIREME).

\section{SOCIAL PARTICIPATION, A FACTOR TO CONSIDER IN THE CLINICAL EVALUATION OF THE OLDER ADULT: A NARRATIVE REVIEW}

\begin{abstract}
Social participation is defined as the integration of people into community activities in voluntary or mandatory, formal and informal social groups, which could have consequences concerning the health of the older population. A search was conducted on Pubmed, Scielo, Scopus and Google Scholar. For this review, 16 articles studying the impact of social participation on older adults were analyzed, including a total of 73,698,096 individuals from North America, Asia, Europe, and Latin America. Social participation is considered a protective factor for the mental and physical health of older adults, which has been associated with decreases in disability, co-morbidities, and mortality. For this reason, it is suggested that it should be evaluated in clinical practice. This would make it possible to orient and refer older adults to participate in certain community organizations, mainly those who do not have support networks, who are not linked to community groups, who have symptoms of depression or who are beginning to show physical or cognitive deterioration. In this way, public health could increase its prevention and health promotion actions through community organizations. On the other hand, the lack of instruments and consensus to evaluate social participation was discussed in this review where a questionnaire to evaluate the social participation of the older adult has been proposed to be validated and studied in the future.
\end{abstract}

Keywords: Social Participation; Aging; Functionality; Health. (source: MeSH NLM).

\section{INTRODUCCIÓN}

El envejecimiento es un proceso caracterizado por un decline en la capacidad funcional relacionado a disminución de la fuerza muscular, equilibrio, capacidad aeróbica, flexibilidad y también algunas funciones cognitivas, como la memoria ${ }^{(1,2)}$. La funcionalidad del adulto mayor se ve afectada 
por factores intrínsecos como la prevalencia de algunas enfermedades crónicas y factores extrínsecos como el tipo de vivienda, el nivel educacional, el estado socioeconómico y la participación social ${ }^{(3,4)}$. El equilibrio entre estos factores son determinantes para la salud y la calidad de vida de los adultos mayores ${ }^{(5-7)}$.

La Organización Mundial de la Salud describe una estrecha relación entre las funciones físicas con el nivel de autonomía y de participación social en la comunidad ${ }^{(8)}$. Respecto de la participación social, esta ha sido definida como la participación activa en organizaciones comunitarias de carácter religioso, deportivo, cultural, recreativos, políticas y de voluntariados ${ }^{(9-12)}$. Diversos estudios han reportado efectos protectores de la participación social para la salud del adulto mayor, siendo considerada como un estímulo para aumentar el nivel de actividad física y la capacidad de sociabilización, lo que favorece las funciones cognitivas ${ }^{(9,13)}$.

A pesar de que la baja participación social ha sido asociada a problemas de salud y discapacidad en el adulto mayor ${ }^{(9,14-16)}$, es necesario conocer el impacto real sobre la salud y funcionalidad en esta población. En la literatura se han definido algunos métodos para medir el nivel de participación social ${ }^{(9,10,17,18)}$, sin embargo, existen escasos instrumentos como cuestionarios que la evalúen de manera integral y que puedan ser utilizados en el área clínica ${ }^{(17,19)}$.

Por todo lo dicho, esta revisión narrativa tuvo como objetivo analizar el efecto de la participación social en la salud de las personas adultas mayores e identificar los principales instrumentos de evaluación de la participación social.

\section{METODOLOGÍA}

La búsqueda de la literatura fue realizada por dos autores (WSL y RSL). Fueron consultadas las siguientes bases de datos Pubmed, Scielo, Scopus y Google Scholar usando los siguientes términos MeSH («social participation» and «aging») y DeCS («participación social» y «envejecimiento»). Fueron incluidos artículos de investigación de cualquier tipo de diseño metodológico publicados desde el 2010 hasta el 2020, en los idiomas inglés, español o portugués. Se excluyeron los artículos que no describían en la metodología los criterios para definir la participación social y también aquellos cuya población de estudio fuera menor de 55 años.

Las listas de artículos de las bases de datos fueron bajadas en «formato bib» y almacenadas en el programa STAR (State of the Art through Systematic Review) y en Mendeley para analizar artículos duplicados, lecturas de títulos y resumenes. El contenido de la revisión fue dividido en tres áreas principales: 1) definición de la participación social; 2) beneficios de la participación social para la salud física y mental del adulto mayor; 3) salud pública y participación social; y 4) evaluación de la participación social.

\section{RESULTADOS Y DISCUSIÓN}

Después de analizar duplicaciones, la lectura de títulos y resúmenes, y de pasar por los criterios de exclusión, fueron escogidos 16 estudios sobre participación social en adultos mayores y sus efectos para la salud. En la Tabla 1 se presentan las características de los estudios seleccionados para la revisión. Los estudios seleccionados son en su mayoría de diseño longitudinal e incluyen un total de 73698096 adultos mayores de Norteamérica (6 artículos), Asia (5 artículos), Europa ( 3 artículos), y América latina ( 2 artículos). Además de los artículos seleccionados hemos incluido otros complementarios para la discusión de los resultados.

\section{DEFINICIÓN DE LA PARTICIPACIÓN SOCIAL}

De acuerdo con Desley, et al ${ }^{(20)}$ y Loyola, et al ${ }^{(9)}$, la participación social es un indicador individual de la integración de las personas a actividades de la comunidad en grupos específicos de carácter formal o informal que genera interacciones sociales con otros individuos. En este sentido, la participación social es considerada uno de los factores protectores para la salud y la funcionalidad del adulto mayor, junto con el estilo de vida, el apoyo familiar, el nivel educacional, función física y mental, apoyo económico, estado y tipo de enfermedad ${ }^{(9,16,21)}$ (Figura 1).

No existe un consenso sobre los criterios para identificar la participación social en los adultos mayores (Tabla 1). Por ejemplo, de acuerdo con Chan, et al ${ }^{(22)}$, la participación social se relaciona más con las redes de apoyo que puede tener un adulto mayor en la familia o en la comunidad, pero no se describe estrictamente como la vinculación a alguna organización comunitaria como fue planteado por Aida, et al ${ }^{(23)}$. Actualmente se ha ampliado un poco más el concepto de participación social incluyéndose también los factores sociales ${ }^{(24,25)}$, la frecuencia de participación en días, semanas o meses ${ }^{(26)}$ y el rol dentro de una organización o grupo comunitario ${ }^{(26)}$.

Debido a que la participación social puede variar según el tipo y el acceso a actividades en un determinado territorio, la mayoría de los estudios evalúan determinadas actividades según la realidad local (Tabla 1). Por eso, podemos encontrar estudios que consideran actividades de recreación, de lectura, salidas al cine y de cultura, como en el estudio de Facourt, et al ${ }^{(26)}$ en Inglaterra o en el de Tomioka, et al en Japón ${ }^{(27)}$, las cuales que no son de común acceso para los adultos mayores de Latinoamérica.

Entre las organizaciones consideradas por la literatura, existen dos grandes divisiones, las voluntarias y las obligatorias, donde el individuo puede desarrollar el liderazgo o ser un miembro más del grupo ${ }^{(28)}$. Dicho esto, podemos subdividir estas actividades de la siguiente manera: 1) actividades informales de conexión social (visitar la familia, 
amigos o vecinos); 2) actividades sociales en público (salir a restaurantes, clubes de baile, cines, teatros, etc.); 3) actividades sociales de ejercicio o recreación (salir a jugar futbol, ir al gimnasio, asistir a clases de baile, de pintura, etc.); 4) actividades sociales de carácter cívico (asistir a juntas de vecinos, actividades del consejo comunal, reuniones de partidos políticos, etc.); 5) actividades comunitarias (como partici- par de grupos étnicos, grupos religiosos, grupos de adultos mayores, etc.) ${ }^{(28)}$. Otra clasificación más simple es dividir los tipos de organizaciones en verticales y horizontales ${ }^{(29,30)}$, de las cuales los verticales serían grupos políticos, religiosos, juntas de vecinos y asociaciones de adultos mayores; y los horizontales serían grupos voluntarios, deportivos y de recreación.

Tabla 1. Características de los principales estudios incluidos en la revisión

\begin{tabular}{|c|c|c|c|c|c|c|c|}
\hline Autor & Tipo de estudio & País & Muestra, $n$ & $\begin{array}{l}\text { Género, } \mathbf{n} \\
(\mathrm{M} / \mathrm{F})\end{array}$ & Edad & Definición y criterios de participación social & Resultado principal \\
\hline $\begin{array}{l}\text { Chan, et al. } \\
(2011)^{(22)}\end{array}$ & Transversal & Singapur & 4489 & $2078 / 2411$ & $\geq 60$ años & $\begin{array}{l}\text { Ser clasificado entre uno de los } 5 \text { criterios de Lubben } \\
\text { modificado: (1) viviendo con cónyuge (sin hijo); ( } 2) \\
\text { viviendo con un cónyuge y al menos un hijo; (3) viviendo } \\
\text { con al menos un hijo (sin cónyuge); (4) viviendo solo o } \\
\text { con una trabajadora doméstica y (5) viviendo solo con } \\
\text { otras personas (otras incluían a una persona que no era su } \\
\text { esposo o hijo, por ejemplo, padre, hermano, etc.). }\end{array}$ & $\begin{array}{c}\text { Vivir solo y tener pocas } \\
\text { redes de apoyo aumenta } \\
\text { los síntomas depresivos de } \\
\text { hombres y mujeres mayores, } \\
\text { las redes sociales débiles son } \\
\text { especialmente perjudiciales } \\
\text { para los hombres que viven } \\
\text { solos. }\end{array}$ \\
\hline $\begin{array}{l}\text { Aida, } \text { et al. } \\
(2013)^{(23)}\end{array}$ & Prospectivo & Japón & 14589 & $6953 / 7636$ & $\geq 60$ años & $\begin{array}{l}\text { Participación en algún grupo u organización comunitaria } \\
\text { de cualquier interés. }\end{array}$ & $\begin{array}{l}\text { La baja participación social } \\
\text { está asociada con una mayor } \\
\text { incidencia de discapacidad } \\
\text { funcional principalmente en } \\
\text { mujeres. }\end{array}$ \\
\hline $\begin{array}{l}\text { Crittenden, et al. } \\
(2014)^{(31)}\end{array}$ & Longitudinal & EUA & 1147 & $518 / 629$ & 70-79 años & $\begin{array}{l}\text { Participar } 1 \text { vez por mes en actividades sociales: religiosas, } \\
\text { clubes de adultos mayores, voluntariado, trabajo, familia } \\
\text { y amigos. }\end{array}$ & $\begin{array}{l}\text { Participar en actividades } \\
\text { sociales está relacionado con } \\
\text { una mejor función pulmonar. }\end{array}$ \\
\hline $\begin{array}{l}\text { Ferrano, et al. } \\
(2014)^{(32)}\end{array}$ & Longitudinal & EUA & 1693 & $830 / 863$ & $55-85$ años & $\begin{array}{l}\text { Haber participado en algún grupo religioso en los últimos } \\
12 \text { meses. }\end{array}$ & $\begin{array}{l}\text { Participar en grupos religiosos } \\
\text { es más beneficioso para } \\
\text { personas afrodescendientes } \\
\text { que para las personas } \\
\text { blancas. Relacionado con la } \\
\text { disminución de proteína C } \\
\text { reactiva. }\end{array}$ \\
\hline $\begin{array}{l}\text { Choi, et al. } \\
(2015)^{(33)}\end{array}$ & Longitudinal & EUA & 2845 & Solo hombres & $>65$ años & $\begin{array}{l}\text { Participación en el último mes en } 5 \text { actividades: } \\
\text { (1) trabajo remunerado (por cuenta ajena o por cuenta } \\
\text { propia); (2) voluntariado; (3) actividades grupales } \\
\text { formales (participación en clubes, clases u otras } \\
\text { actividades organizadas, no incluidos los servicios } \\
\text { religiosos); (4) asistencia al servicio religioso; y (5) } \\
\text { salidas para disfrutar (por ejemplo, salir a cena, una } \\
\text { película, jugar, escuchar música o ver un jugar). }\end{array}$ & $\begin{array}{l}\text { Las actividades grupales } \\
\text { formales y de recreación se } \\
\text { asociaron positivamente con } \\
\text { un mejor estado de salud } \\
\text { autorreportado. }\end{array}$ \\
\hline $\begin{array}{l}\text { Crezen, et al. } \\
(2015)^{(34)}\end{array}$ & Longitudinal & $\begin{array}{l}\text { Países de } \\
\text { Europa }\end{array}$ & 9068 & $4072 / 4996$ & $>55$ años & $\begin{array}{c}\text { Participación en el último mes en } 5 \text { actividades diferentes: } \\
\text { (1) trabajo voluntario o de caridad; (2) cursos educativos o } \\
\text { de capacitación; ( } 3 \text { ) deportes, clubes sociales u otros tipos } \\
\text { de actividades del club; (4) participación en organizaciones } \\
\text { religiosas; y (5) participación en organizaciones políticas } \\
\text { o comunitarias. }\end{array}$ & $\begin{array}{l}\text { Los efectos de la participación } \\
\text { social en los síntomas de } \\
\text { la depresión dependen del } \\
\text { tipo de actividad. Participar } \\
\text { de actividades religiosas y } \\
\text { espirituales se relaciona con } \\
\text { menos síntomas de depresión. }\end{array}$ \\
\hline $\begin{array}{l}\text { Bourassa, et al. } \\
(2015)^{(35)}\end{array}$ & Longitudinal & $\begin{array}{l}\text { Países de } \\
\text { Europa }\end{array}$ & 19832 & $9381 / 10451$ & $>55$ años & $\begin{array}{c}\text { Participación en el último mes en } 4 \text { actividades: (1) trabajo } \\
\text { voluntario o de caridad; (2) deporte, social u otro tipo de } \\
\text { club; (3) organización religiosa; y (4) una organización } \\
\text { política o comunitaria. }\end{array}$ & $\begin{array}{l}\text { La participación social es un } \\
\text { predictor de funcionamiento } \\
\text { cognitivo a lo largo del tiempo } \\
\text { en un seguimiento de } 8 \text { años } \\
\text { (2004-2012). }\end{array}$ \\
\hline $\begin{array}{l}\text { Gallardo-Peralta, } \\
\text { et al. } \\
(2016)^{(36)}\end{array}$ & Transversal & Chile & 777 & $289 / 488$ & $\geq 60$ años & $\begin{array}{l}\text { Participación en el último mes en } 5 \text { tipos de } \\
\text { organizaciones: (1) clubes de adultos mayores; (2) grupos } \\
\text { religiosos; (3) grupos del barrio (en especial juntas } \\
\text { de vecinos); (4) grupos indígenas y (5) otros grupos } \\
\text { (culturales, deportivos, recreativos, etc.). }\end{array}$ & $\begin{array}{l}\text { El envejecimiento exitoso se } \\
\text { relaciona con mayores niveles } \\
\text { de participación. }\end{array}$ \\
\hline $\begin{array}{l}\text { Tamioka, et al. } \\
(2016)^{(37)}\end{array}$ & Longitudinal & Japón & 6093 & $2768 / 3325$ & $\geq 60$ años & $\begin{array}{c}\text { Participación actual de uno de los siguientes } 5 \text { grupos } \\
\text { comunitarios: (1) juntas de vecinos; (2) grupos de hobby; } \\
\text { (3) grupos de eventos locales; (4) de adultos mayores y (5) } \\
\text { voluntariados. }\end{array}$ & $\begin{array}{l}\text { Ser activo en varios grupos } \\
\text { sociales está asociado con } \\
\text { mantención de la función } \\
\text { cognitiva en mujeres, pero no } \\
\text { en hombres. }\end{array}$ \\
\hline
\end{tabular}

M: masculino; F: femenino; n: número de sujetos.

EUA: Estados Unidos de América 
Tabla 1. Características de los principales estudios incluidos en la revisión (continuación).

\begin{tabular}{|c|c|c|c|c|c|c|c|}
\hline Autor & Tipo de estudio & País & Muestra, $\mathbf{n}$ & $\begin{array}{c}\text { Género, } \mathbf{n} \\
(\mathrm{M} / \mathrm{F})\end{array}$ & Edad & Definición y criterios de participación social & Resultado principal \\
\hline $\begin{array}{l}\text { Cheng, et al. } \\
(2017)^{(38)}\end{array}$ & Transversal & Singapur & 4169 & $1949 / 2220$ & $\geq 60$ años & $\begin{array}{l}\text { Ser clasificado entre uno de los } 5 \text { criterios de Lubben } \\
\text { modificado: (1) viviendo con cónyuge (sin hijo); } \\
\text { (2) viviendo con un cónyuge y al menos un hijo; }(3) \\
\text { viviendo con al menos un hijo (sin cónyuge); (4) } \\
\text { viviendo solo o con una trabajadora doméstica y (5) } \\
\text { viviendo solo con otras personas (otras incluían a } \\
\text { una persona que no era su esposo o hijo, por ejemplo, } \\
\text { padre, hermano, etc.). }\end{array}$ & $\begin{array}{c}\text { Estar casado y tener redes } \\
\text { sociales más fuertes puede } \\
\text { amortiguar el impacto cognitivo } \\
\text { negativo de la duración extrema } \\
\text { del sueño. }\end{array}$ \\
\hline $\begin{array}{l}\text { Loyola, et al. } \\
(2017)^{(9)}\end{array}$ & Transversal & Chile & 164 & $37 / 127$ & $\geq 60$ años & $\begin{array}{l}\text { Participar de una organización comunitaria al menos } \\
\text { una vez por semana (cualquier tipo de organización } \\
\text { comunitaria formal o informal). }\end{array}$ & $\begin{array}{c}\text { Individuos con baja } \\
\text { participación social tienen más } \\
\text { comorbilidades, discapacidad y } \\
\text { menos fuerza muscular. }\end{array}$ \\
\hline $\begin{array}{l}\text { Tamioka, et al. } \\
(2017)^{(27)}\end{array}$ & Longitudinal & Japón & 6360 & $2774 / 3586$ & 65-96 años & $\begin{array}{l}\text { Participar de una de las siguientes actividades: } \\
\text { (1) eventos locales (por ejemplo, festivales); (2) } \\
\text { asociaciones comunitarias de vecindarios; (3) clubes } \\
\text { de pasatiempos; (4) clubes de adultos mayores y (5) } \\
\text { grupos de voluntarios. }\end{array}$ & $\begin{array}{l}\text { Participar de diferentes tipos } \\
\text { de actividades sociales previene } \\
\text { el declino de las actividades } \\
\text { instrumentales de la vida diaria } \\
\text { en el tiempo. }\end{array}$ \\
\hline $\begin{array}{l}\text { Facourt, et al. } \\
(2018)^{(26)}\end{array}$ & Longitudinal & Inglaterra & 2548 & no clarificado & $\geq 55$ años & $\begin{array}{l}\text { Participación en uno de los } 8 \text { grupos comunitarios: } \\
\text { (1) partidos políticos, sindicatos o grupos } \\
\text { ambientalistas; (2) grupos de inquilinos, grupos de } \\
\text { residentes, grupos de vigilancia del vecindario; (3) } \\
\text { iglesia u otros grupos religiosos; (4) asociaciones } \\
\text { de caridad; (5) grupos de educación, artes o música } \\
\text { o clases nocturnas; (6) clubes sociales; (7) clubes } \\
\text { deportivos, gimnasios, clases de ejercicio; y (8) } \\
\text { cualquier otra organización, club o sociedad. }\end{array}$ & $\begin{array}{l}\text { Ser miembro de } 2 \text { tipos de } \\
\text { grupos comunitarios, como } \\
\text { de educación, artes, música } \\
\text { o religión puede apoyar el } \\
\text { bienestar en la vejez. }\end{array}$ \\
\hline $\begin{array}{l}\text { Rotenberg, et al } \\
(2020)^{(24)}\end{array}$ & Transversal & Canadá & 115 & $41 / 74$ & $\geq 60$ años & $\begin{array}{c}\text { Fue utilizado el Activity Card Sort, una medición } \\
\text { autorreportada que examina las actividades de } \\
\text { participación en el último tiempo, y que divide } \\
\text { las actividades en } 4 \text { dominios: } 1 \text { ) actividades } \\
\text { instrumentales de la vida diaria; } 2 \text { ) actividades de } \\
\text { ocio con bajas demandas físicas; } 3 \text { ) actividades de } \\
\text { ocio con altas demandas físicas; y 4) actividades } \\
\text { sociales. }\end{array}$ & $\begin{array}{l}\text { La participación social está } \\
\text { relacionada con buen estado } \\
\text { cognitivo y memoria. }\end{array}$ \\
\hline $\begin{array}{l}\text { Bernier, et al } \\
(2020)^{(25)}\end{array}$ & Transversal & Canadá & 2787 & $1142 / 1645$ & $\geq 65$ años & $\begin{array}{l}\text { Evaluado por medio de un cuestionario creado } \\
\text { para el proyecto Seniors Health Survey, que cuenta } \\
\text { con } 12 \text { preguntas que miden diversos aspectos } \\
\text { sociales (no solamente la participación social). Las } \\
\text { respuestas de algunas preguntas son «sí» o «no», y } \\
\text { en otras 1) casi siempre, 2) la mitad del tiempo y } \\
\text { 3) casi nunca. }\end{array}$ & $\begin{array}{l}\text { Participar de organizaciones } \\
\text { disminuye el deseo de morir. }\end{array}$ \\
\hline
\end{tabular}

M: masculino; F: femenino; n: número de sujetos.

\section{BENEFICIOS DE LA PARTICIPACION SOCIAL PARA LA SALUD FÍSICA Y MENTAL DEL ADULTO MAYOR}

Diversos son los beneficios de la participación social para la salud mental y física de los adultos mayores que han sido descritos por la literatura ${ }^{(9,13,20,39,40)}$. En relación con la función mental, las actividades sociales ayudan a preservar la capacidad cognitiva en la población adulta mayor ${ }^{(39)}$, debido a que la participación social es un estímulo que mantiene al adulto mayor vinculado con el medio y la comunidad. Al participar en reuniones, el adulto mayor se mantiene conectado en el tiempo y el espacio, así también, interactúa con otros adultos mayores, lo cual estimula los sistemas sensoriales, la autoestima, la afectividad, y el apoyo emocional y psicológico ${ }^{(9,13,41)}$ (Figura 2).

De acuerdo con Chan, et al ${ }^{(22)}$, vivir solo y tener pocas redes de apoyo aumenta los síntomas depresivos entre hombres y mujeres mayores. La participación social disminuye los síntomas depresivos y mejora la capacidad cognitiva, según un estudio de seguimiento de 8 años por Bourassa, et al ${ }^{(35)}$. En este sentido, Bernier, et al ${ }^{(25)}$ reportaron que la participación social sería un factor protector que disminuye el deseo de suicidio de los adultos mayores, esto puede ser explicado por los efectos antidepresivos de la participación social descritos previamente por Crezen, et al ${ }^{(34)}$. Este mismo autor señala que participar de cualquier grupo comunitario genera efectos beneficiosos para la salud emocional de la persona mayor. Sin embargo, los más grandes beneficios emocionales se producen cuando los adultos mayores se vinculan a organizaciones religiosas o espirituales ${ }^{(34)}$.

Un estudio prospectivo realizado por Tomioka, et al (27) constató que la participación social en diferentes actividades estaría asociado con un aumento en las actividades de la vida diaria en mujeres. De acuerdo con este estudio, los hallazgos resaltan la importancia de incentivar a los adultos mayores a participar en actividades sociales para mantener la funcionalidad en las actividades de la vida diaria. Por lo que participar de reuniones o actividades sociales es un estímulo que aumenta el nivel de actividad física, el cual es un factor importante para 


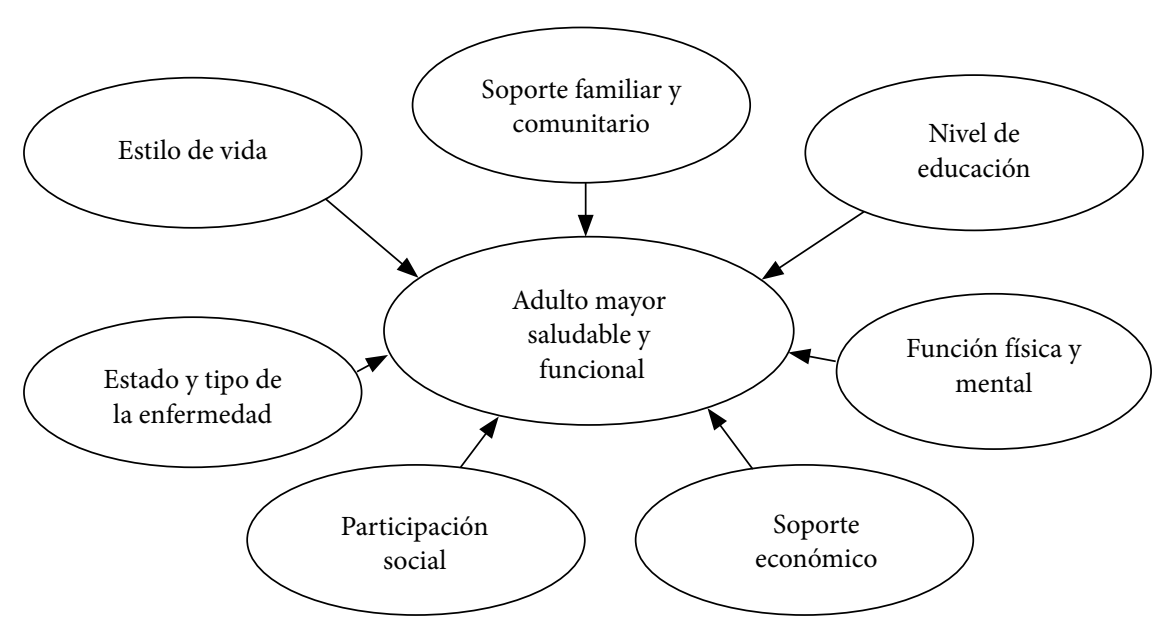

Figura 1. Principales factores que determinan la funcionalidad y el estado de salud de un adulto mayor

la salud de las personas de la tercera edad ${ }^{(42)}$ (Figura 2). Este aumento de la actividad física puede generar efectos no solo en la capacidad funcional, sino que también a nivel sistémico ${ }^{(43,44)}$. Esto puede ayudar a comprender la relación entre participación social y buena función pulmonar, reportadas por Crittenden, et al ${ }^{(31)}$ o con baja inflamación sistémica, observada por Ferrano, et al en individuos adultos mayores que estaban vinculados a actividades comunitarias ${ }^{(32)}$. Por esta razón, los adultos mayores que son socialmente activos presentan mayor funcionalidad, más hábitos saludables, menor número de comorbilidades y bajo riesgo de mortalidad que aquellos que no participan de organizaciones comunitarias ${ }^{(9,13-16,33,45)}$. De esta manera, como fue descrito por Gallardo-Peralta, et al ${ }^{(36)}$, el envejecimiento exitoso se relaciona con mayores niveles de participación.
Es importante considerar que los efectos de la participación social sobre la salud de los adultos mayores dependen del tipo de actividad social, localidad (urbano o rural), raza, edad y género $^{(41,46,48)}$. Por ejemplo, las actividades espirituales o religiosas se han asociado más a reducciones en síntomas depresivos en adultos mayores comparado a otros tipos de actividades ${ }^{(34)}$. Según Hernández-Huayta, et al los adultos mayores que viven en zonas rurales tienen una mayor participación social y comunitaria que aquellos que viven en zonas urbanas ${ }^{(46)}$. Adicionalmente, mayores efectos de la participación social han sido encontrados en mujeres comparado a los hombres ${ }^{(37)}$, donde, por lo general, las mujeres participan más activamente que los hombres en organizaciones comunitarias ${ }^{(9,41)}$.

Por otra parte, existen algunos tipos de participación, ya descritos, que no siempre tienen efectos positivos ${ }^{(49)}$, como

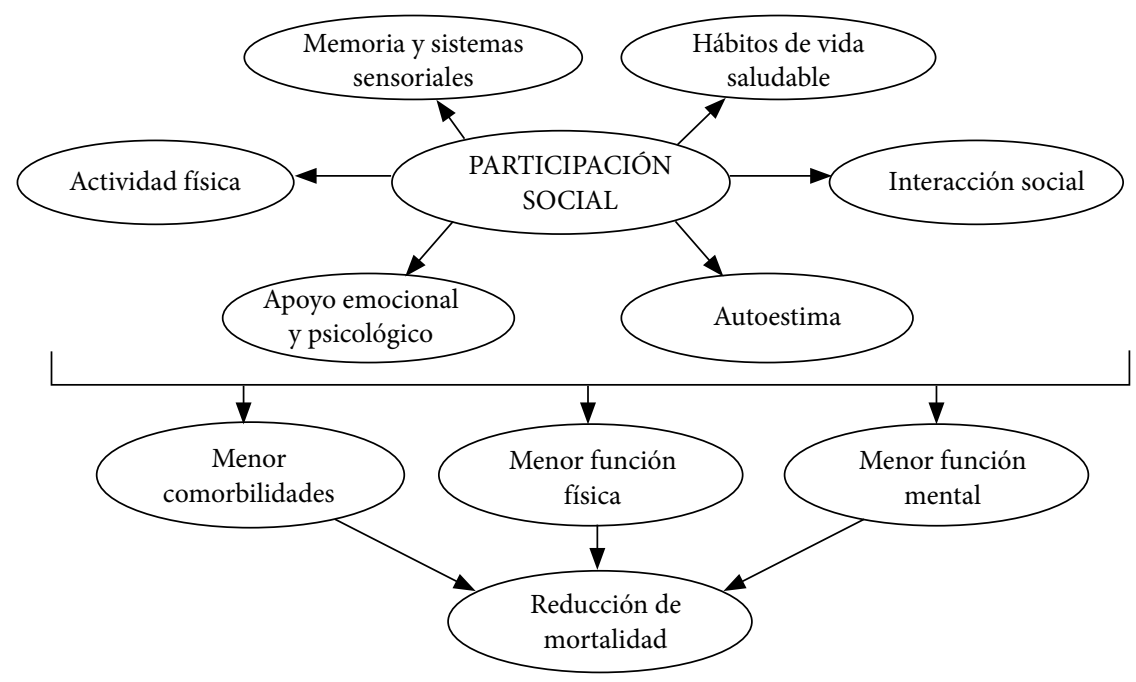

Figura 2. Principales efectos de la participación social para la salud del adulto mayor. La participación social contribuye a una mejor función física, mental y menor comorbilidades, que llevan a una reducción de la mortalidad del adulto mayor. 
aquellas actividades obligatorias, las cuales puede tener efectos negativos en la salud mental de esta población ${ }^{(49)}$. Por este motivo, se recomienda que los adultos mayores realicen actividades que estimulen su participación de manera autónoma ${ }^{(49)}$, a fin de lograr una vida activa y saludable ${ }^{(41)}$.

\section{SALUD PÚBLICA Y PARTICIPACIÓN SOCIAL}

Actualmente existen algunos programas que han incentivado el empoderamiento de los adultos mayores en la sociedad ${ }^{(50-52)}$. La participación social ha sido fuertemente estudiada en países como Japón, Estados Unidos y Canadá ${ }^{(24,31,41)}$, donde se destacan algunas estrategias para aumentar la participación social, como la disponibilidad de espacios comunitarios para actividades de desarrollo humano, artístico, cultural, deportivo, espiritual, cívico y recreativo ${ }^{(26,33)}$. En Latinoamérica, si bien existen programas propuestas por los ministerios de salud; la participación social especialmente de los adultos mayores ha sido poco estudiada ${ }^{(9,36)}$.

En Brasil, se creó la Política Nacional para Personas Mayores, promulgada en 1994 y regulada en 1996, que garantiza los derechos sociales de las personas mayores, creando condiciones para promover su autonomía, integración y participación efectiva en la sociedad y reafirmando el derecho a la salud en diferentes niveles de salud ${ }^{(53)}$. Uno de los objetivos es garantizar la atención a toda la población, dar visibilidad a las personas mayores con un alto grado de dependencia funcional, crear entornos físicos, sociales y actitudinales que permitan mejorar la salud de las personas con discapacidad, con el objetivo de ampliar su participación social en la comunidad ${ }^{(53)}$.

En Chile, un enfoque basado en los derechos ha cobrado impulso en las políticas públicas y sociales sobre el envejecimiento ${ }^{(52)}$. El objetivo subyacente es proporcionar garantías para el bienestar integral de la población adulta mayor. Unas de las estrategias de participación que impulsó el Ministerio de Salud de Chile para los adultos mayores fue el Programa Más Adultos Mayores Autovalentes, que no solo fomenta la actividad física, sino también la participación social e integración a actividades comunitarias ${ }^{(50)}$. Los adultos mayores participan principalmente en organizaciones sociales religiosas, consejos vecinales y grupos de adultos mayores ${ }^{(36)}$. De esta manera, a pesar de las iniciativas de Ministerio de Salud chileno, la participación es relativamente baja y el sistema sanitario no consigue atender una gran parte de la población ${ }^{(54)}$.

En el Perú, se formó una comisión multisectorial para formular el Plan Nacional para las Personas Adultas Mayores (PLANPAM) ${ }^{(55)}$ cuyo objetivo fue mejorar la calidad de vida de los adultos mayores; todo basado en cuatro políticas nacionales esenciales: a) envejecimiento saludable; b) empleo, bienestar y seguridad social; c) educación, conciencia y cultura sobre el envejecimiento y la vejez; y d) participación e integración social. El Plan consideró grupos para adultos mayores, actividad física, consejos locales de salud y consejos comunitarios donde los adultos mayores pueden ser escuchados y presentar sus demandas y prioridades. La participación social en adultos mayores peruanos a pesar de estar presente como una de las prioridades del PLANPAM, ha sido poco estudiada, lo cual puede ser relacionado a la falta de instrumentos de evaluación y capacitación en el área ${ }^{(56)}$.

En este sentido Hernández-Huayta, et al ${ }^{(46)}$ en su estudio evalúa la participación social en adultos mayores peruanos, sin embargo, el instrumento que utilizan, de acuerdo con los autores, no ha sido validado en población peruana. Esta falta de instrumentos de evaluación de la participación social acontece también en otros países de Latinoamérica y del mundo. De acuerdo con Soto, et al ${ }^{(56)}$ son diversos los desafíos para la investigación en salud pública para la realidad latinoamericana, por lo que el estudio de la participación social y sus efectos en la salud de las personas adultas mayores será probablemente un foco importante para los investigadores en el área de la gerontología y la salud pública.

\section{EVALUACIÓN DE LA PARTICIPACIÓN SOCIAL}

A pesar de las evidencias actuales sobre los efectos de la participación social para la salud del individuo, existen escasos instrumentos que la evalúen de una manera integral ${ }^{(17)}$. La participación social ha sido evaluada dentro de subítems inmersos en otros cuestionarios no específicos ${ }^{(19,25)}$, o por medio de preguntas simples que miden si el adulto mayor ha participado o no en alguna organización social en un determinado periodo de tiempo ${ }^{(9,10,18,35)}$.

Entre los instrumentos que se usan en la práctica clínica para evaluar la participación social, los más utilizados son: Community Integration Questionnaire (CIQ) ${ }^{(57)}$, Participation Scale (P-Scale) ${ }^{(58)}$ y Social Participation Restrictions Questionnaire (SPaRQ) ${ }^{(59)}$. También se ha sugerido cuantificar el nivel de participación social, por medio de escalas de 5 a 10 ítems ${ }^{(26,28,33,35,60,61)}$, que evalúa la frecuencia en días por mes, de diversas actividades sociales, como salir con amigos o familia; actividades religiosas; actividades deportivas; actividades educacionales o culturales; reuniones de clubes del adulto mayor; actividades en asociaciones profesionales, de vecinos o jubilados; actividades voluntarias de caridad y actividades recreacionales o de otra índole.

Debido a la amplia definición de participación social y a la variedad de tipos de actividades que pueden considerarse parte de este concepto, la utilización de preguntas simples ${ }^{(9,26,35,36,60,61)}$ puede ser una buena estrategia de evaluación, considerando que los tipos de organizaciones o grupos comunitarios, y las oportunidades de participación social pueden variar en cada país. En este sentido, en la literatura se han reportado dos pun- 
tos de corte para definir la baja participación social ${ }^{(9,17)}$, que pueden ser utilizados en la evaluación del adulto mayor de una manera rápida y objetiva. Estos puntos de corte dependen del número de veces que una persona participa en algún tipo de organización o grupo comunitario, el primero es «participar menos de una vez por semana» y está asociado con mayor discapacidad ${ }^{(9)}$, y el segundo es «participar menos de una vez por mes» y está relacionado con mayor mortalidad ${ }^{(17)}$.

La participación social, como se demostró en esta revisión, está relacionada con la salud y funcionalidad del adulto mayor $^{(9,13,20,26,35,36,39,40,60,61)}$. De este modo, la elección del criterio de evaluación puede depender de diferentes factores que el profesional del área de salud debe tener en consideración, como el tiempo de evaluación y los tipos de organizaciones comunitarias disponibles para el adulto mayor en un determinado país.

En relación con lo anterior, los investigadores del presente estudio han propuesto una ficha de evaluación con un cuestionario sobre la base de la literatura analizada, que puede ser utilizada para la evaluación de la participación social de los adultos mayores para ser aplicado en los sistemas de salud pública en Latinoamérica.

El cuestionario considera 6 diferentes tipos de actividades relacionadas a la participación social del adulto mayor: (1) actividades o reuniones con organizaciones de adultos mayores; (2) actividades o reuniones de junta de vecinos o partido político; (3) actividades o reuniones religiosas, de crecimiento personal o espiritual; (4) actividades familiares o con amigos; (5) actividades de voluntariado para ayudar a otras personas; (6) actividades recreativas en la comunidad, como fiestas, actividades deportivas, culturales, musicales asociadas a algún otro pasatiempo.

Las opciones de respuesta del adulto mayor a la pregunta "¿has participado de alguna de las 6 actividades señaladas?» son las siguientes: 1) No, nunca; 2) sí, una vez por mes; 3) sí, de 2 a 4 veces por mes; 4) sí, una vez por semana; 5) sí, más de una vez por semana; con puntuaciones de 0 a 4 respectivamente. Además, se consideró evaluar la función que tiene el adulto mayor en la organización o en el grupo que participa con la pregunta « ¿formas parte de la directiva de alguna de las organizaciones o grupos de los cuales participas?».

\section{REFERENCIAS BIBLIOGRÁFICAS}

1. Tuna HD, Edeer AO, Malkoc M, Aksakoglu G. Effect of age and physical activity level on functional fitness in older adults. Eur Rev Aging Phys Act. 2009;6(2):99-106

2. Rolland Y, Czerwinski S, Abellan Van Kan G, Morley JE, Cesari M, Onder G, et al. Sarcopenia: its assessment, etiology, pathogenesis, consequences and future perspectives. J Nutr Health Aging [Internet]. 2011;12(7):433-50. Available from: http://www.ncbi.nlm.nih.gov/pubmed/18615225\%0A http://www.pubmedcentral.nih.gov/articlerender. fcgi?artid=PMC3988678.
El cuestionario de participación social del adulto mayor (Anexo 1) será útil para identificar los tipos de participación que los adultos mayores están realizando y poder intervenir tempranamente en aquellos adultos mayores que no tienen redes de apoyo comunitario. Adicionalmente, futuros estudios podrían validar el cuestionario propuesto en esta revisión y su relación con la funcionalidad física y mental de los adultos mayores de Latinoamérica.

\section{CONCLUSIÓN}

La participación social puede ser definida como la vinculación voluntaria u obligatoria a una organización o grupo social formal e informal. Es un factor protector para la salud mental y física del adulto mayor que debería ser evaluada en la práctica clínica por medio de preguntas simples o cuestionarios, debido al gran impacto que tiene la participación social en la salud de los adultos mayores. La evaluación permitiría orientar y derivar a los adultos mayores a participar en determinadas organizaciones comunitarias, principalmente aquellos que no están vinculados en redes de apoyo o que no forman parte de algún grupo comunitario y que están teniendo síntomas de depresión o iniciando un cuadro de deterioro físico o cognitivo. Esto ampliaría aún más los horizontes de la salud pública, vinculado las acciones de prevención y promoción de la salud con las organizaciones comunitarias.

Finalmente, más investigaciones son necesarias, principalmente en Latinoamérica, donde hay poca evidencia disponible, para identificar los mecanismos específicos que explican la asociación entre participación social y salud de los adultos mayores, así como la elaboración y validación de más instrumentos de evaluación que puedan ser utilizados en la práctica clínica.

Contribuciones de autoría: WASL y RDSL han participado en la búsqueda de literatura, y en el diseño y construcción del artículo. RPTM y VSP participaron en la redacción, así como aprobación de la versión final.

Fuentes de financiamiento: Autofinanciado.

Conflictos de interés: Los autores declaran no tener conflictos de interés en la publicación del artículo.

Material suplementario: Disponible en la versión electrónica de la RPMESP.

3. Cruz-Jentoft AJ, Baeyens JP, Bauer JM, Boirie Y, Cederholm T, Landi F, et al. Sarcopenia: European consensus on definition and diagnosis. Age Ageing. 2010;39(4):412-23.

4. Glass TA, de Leon CM, Marottoli RA, Berkman LF. Population based study of social and productive activities as predictors of survival among elderly Americans. BMJ [Internet]. 1999 Aug 21;319(7208):478-83. Available from: http://www.ncbi.nlm.nih.gov/pubmed/28199.

5. Willie-Tyndale D, Holder-Nevins D, Mitchell-Fearon K, James K, Laws H, Waldron NK, et al. Participation in Social Activities and the Association 
with Socio-Demographic and Health-Related Factors among Community-Dwelling Older Adults in Jamaica. J Cross Cult Gerontol [Internet] 2016 Dec;31(4):427-47.

6. Unger JB, McAvay G, Bruce ML, Berkman L, Seeman T. Variation in the impact of social network characteristics on physical functioning in elderly persons: MacArthur Studies of Successful Aging. J Gerontol B Psychol Sci Soc Sci [Internet]. 1999 Sep;54(5):S245-51.

7. Takagi D, Kondo K, Kawachi I. Social participation and mental health: moderating effects of gender, social role and rurality. BMC Public Health [Internet]. 2013;13(1):701.

8. Who. The International Classification of Functioning, Disability and Health. World Heal Organ. 2001;18:237.

9. Loyola WS, Camillo CA, Torres CV, Probst VS. Effects of an exercise model based on functional circuits in an older population with different levels of social participation. Geriatr Gerontol Int. 2017.

10. Sirven N, Debrand T. Social participation and healthy ageing: An international comparison using SHARE data. Soc Sci Med [Internet]. 2008;67(12):201726. doi: http://dx.doi.org/10.1016/j.socscimed.2008.09.056.

11. Pollack CE, von dem Knesebeck O. Social capital and health among the aged: comparisons between the United States and Germany. Health Place [Internet]. 2004;10(4):383-91.

12. Yazawa A, Inoue Y, Fujiwara T, Stickley A, Shirai K, Amemiya A, et al. Association between social participation and hypertension among older people in Japan: The JAGES Study. Hypertens Res [Internet]. 2016;39(11):818-24. doi: http://dx.doi.org/10.1038/hr.2016.78.

13. Douglas H, Georgiou A, Westbrook J. Social participation as an indicator of successful aging: An overview of concepts and their associations with health. Aust Heal Rev. 2017;41(4):455-62.

14. Chiao C, Weng LJ, Botticello AL. Social participation reduces depressive symptoms among older adults: An 18-year longitudinal analysis in Taiwan. BMC Public Health. 2011;11. doi: 10.1186/1471-2458-11-292.

15. Wallace LMK, Theou O, Pena F, Rockwood K, Andrew MK. Social vulnerability as a predictor of mortality and disability: cross-country differences in the survey of health, aging, and retirement in Europe (SHARE). Aging Clin Exp Res. 2015;27(3):365-72.

16. Holt-Lunstad J, Smith TB, Layton JB. Social relationships and mortality risk: A meta-analytic review. PLoS Med. 2010;7(7). doi: 10.1371/ journal.pmed.1000316.

17. Saito M, Kondo K, Ojima T, Hirai H, JAGES group. Criteria for social isolation based on associations with health indicators among older people. A 10-year follow-up of the Aichi Gerontological Evaluation Study. Nihon Koshu Eisei Zasshi [Internet]. 2015;62(3):95-105. doi: 10.11236/ jph.62.3_95.

18. Alizadeh S, Mohseni M, Khanjani N, Momenabadi V.Correlation between social participation of women and their quality of life in Kerman. J Heal Promot Manag [Internet]. 2014;3(2):34-42.

19. Pinto JM, Neri AL. Trajectories of social participation in old age: a systematic literature review. Rev Bras Geriatr Gerontol. 2017;20(2):259-72. doi: http://dx.doi.org/10.1590/1981-22562017020.160077.

20. Densley K, Davidson S, Gunn JM. Evaluation of the Social Participation Questionnaire in adult patients with depressive symptoms using Rasch analysis. Qual Life Res. 2013;22(8):1987-97.

21. Ponce MSH, Rosas RPE, Lorca MBF. Social capital, social participation and life satisfaction among Chilean older adults. Rev Saude Publica. 2014 Oct;48(5):739-49.

22. Chan A, Malhotra C, Malhotra R, Østbye T. Living arrangements, social networks and depressive symptoms among older men and women in Singapore. Int J Geriatr Psychiatry. 2011;26(6):630-9.

23. Aida J, Kondo K, Kawachi I, Subramanian S V, Ichida Y, Hirai H, et al. Does social capital affect the incidence of functional disability in older Japanese? A prospective population-based cohort study. J Epidemiol Community Health. 2013 Jan;67(1):42-7.

24. Rotenberg S, Maeir A, Dawson DR. Changes in Activity Participation Among Older Adults With Subjective Cognitive Decline or Objective Cognitive Deficits. Front Neurol. 2020;10(January):1-8.
25. Bernier S, Lapierre S, Desjardins S. Social Interactions among Older Adults Who Wish for Death. Clin Gerontol. 2020;43(1):4-16.

26. Fancourt D, Steptoe A. Community group membership and multidimensional subjective well-being in older age. J Epidemiol Community Health. 2018 May;72(5):376-82.

27. Tomioka K, Kurumatani N, Hosoi H. Association Between Social Participation and 3-Year Change in Instrumental Activities of Daily Living in Community-Dwelling Elderly Adults. J Am Geriatr Soc. 2017 Jan;65(1):107-13.

28. Baum FE, Bush RA, Modra CC, Murray CJ, Cox EM, Alexander KM, et al. Epidemiology of participation : an Australian community study Epidemiology of participation : an Australian community study. Community Health (Bristol). 2000;(January 2009):414-23.

29. Aida J, Hanibuchi T, Nakade M, Hirai H, Osaka K, Kondo K. The different effects of vertical social capital and horizontal social capital on dental status: A multilevel analysis. Soc Sci Med [Internet]. 2009;69(4):512-8. doi: http://dx.doi.org/10.1016/j.socscimed.2009.06.003.

30. Anaby D, Miller WC, Eng JJ, Jarus T, Noreau L. Participation and well-Being Among Older Adults Living with Chronic Conditions. Soc Indic Res. 2011;100(1):171-83.

31. Crittenden CN, Pressman SD, Cohen S, Janicki-Deverts D, Smith BW, Seeman TE. Social integration and pulmonary function in the elderly. Heal Psychol. 2014;33(6):535-43.

32. Ferraro KF, Kim S. Health benefits of religion among black and white older adults? Race, religiosity, and C-reactive protein. Soc Sci Med. 2014;120:92-9.

33. Choi NG, DiNitto DM, Marti CN. Social participation and self-rated health among older male veterans and non-veterans. Geriatr Gerontol Int. 2016 Aug;16(8):920-7.

34. Croezen S, Avendano M, Burdorf A, van Lenthe FJ. Social participation and depression in old age: a fixed-effects analysis in 10 European countries. Am J Epidemiol. 2015 Jul;182(2):168-76.

35. Bourassa KJ, Memel M, Woolverton C, Sbarra DA. Social participation predicts cognitive functioning in aging adults over time: comparisons with physical health, depression, and physical activity. Aging Ment Heal. 2017;21(2):133-46.

36. Gallardo-Peralta L, Conde-Llanes D, Córdova-Jorquera I. Asociación entre envejecimiento exitoso y participación social en personas mayores chilenas. Gerokomos. 2016;27(3):104-8.

37. Tomioka K, Kurumatani N, Hosoi H. Social Participation and Cognitive Decline among Community-dwelling Older Adults: A Community-based Longitudinal Study. Journals Gerontol - Ser B Psychol Sci Soc Sci. 2018;73(5):799-806.

38. Cheng GH-L, Chan A, Lo JC. Importance of social relationships in the association between sleep duration and cognitive function: data from community-dwelling older Singaporeans. Int psychogeriatrics. 2018 Jun;30(6):893-901.

39. Sakamoto A, Ukawa S, Okada E, Sasaki S, Zhao W, Kishi T, et al. The association between social participation and cognitive function in community-dwelling older populations: Japan Gerontological Evaluation Study at Taisetsu community Hokkaido. Int J Geriatr Psychiatry. 2017 Oct;32(10):1131-40.

40. Owari Y, Miyatake N, Kataoka H. Relationship between Social Participation, Physical Activity and Psychological Distress in Apparently Healthy Elderly People: A Pilot Study. Acta Med Okayama. 2018 Feb;72(1):31-7.

41. Tomioka K, Kurumatani N, Hosoi H. Social Participation and the Prevention of Decline in Effectance among Community-Dwelling Elderly: A Population-Based Cohort Study. PLoS One. 2015;10(9):e0139065.

42. Fernandez-Alonso L, Muñoz-García D, La Touche R. The level of physical activity affects the health of older adults despite being active. J Exerc Rehabil [Internet]. 2016;12(3):194-201. doi: 10.12965/jer.1632566.283.

43. da Silva EM, Sepúlveda-Loyola W, Martins da Silva J, Castilho dos Santos G, Pereira C. Comparación entre simple y doble tarea, capacidad cognitiva y equilibrio postural en adultos mayores que participan de 3 modalidades de ejercicio físico. Fisioterapia [Internet]. 2020;42(1):3338. doi: 10.1016/j.ft.2019.10.002. 
44. Cesari M, Costanzo L, Giua R, Vellas B, Incalzi RA. Physical function and exercise in older patients with cardiovascular and respiratory conditions. Curr Pharm Des [Internet]. 2014;20(19):3198-214.

45. Yazawa A, Inoue Y, Fujiwara T, Stickley A, Shirai K, Amemiya A, et al. Association between social participation and hypertension among older people in Japan: the JAGES Study. Hypertens Res. 2016 Nov;39(11):818-24.

46. Hernandez-Huayta J, Chavez-Meneses S, Carreazo NY. Salud y calidad de vida en adultos mayores de un área rural y urbana del Perú. Rev Peru Med Exp Salud Publica. 2016;33(4):680-8.

47. Willie-Tyndale D, Holder-Nevins D, Mitchell-Fearon K, James K, Laws H, Waldron NK, et al. Participation in Social Activities and the Association with Socio-Demographic and Health-Related Factors among Community-Dwelling Older Adults in Jamaica. J Cross Cult Gerontol. 2016;31(4):427-47.

48. Vogelsang EM. Older adult social participation and its relationship with health: Rural- urban differences Eric. 2016;42(May):111-9.

49. Tomioka K, Kurumatani N, Hosoi H. Positive and negative influences of social participation on physical and mental health among community-dwelling elderly aged 65-70 years: a cross-sectional study in Japan. BMC Geriatr. 2017 Dec;17(1):111.

50. Ministerio de Salud de Chile. Orientación técnica programa más adultos mayores autovalentes. 2015. Disponible en: http://www.bibliotecaminsal. cl/wp/wp-content/uploads/2018/01/013.Orientaci\%C3\%B3n-T\%C3\%A9cnica-Programa-Ms-Autovalentes.pdf.

51. Andrade KA De, Toledo MTT De, Lopes MS, Carmo GES Do, Lopes ACS. Aconselhamento sobre modos saudáveis de vida na Atenção Primária e práticas alimentares dos usuários. Rev Esc Enferm USP [Internet]. 2012;46(5):1117-24.

52. Engler T. El empoderamiento de adultos mayores organizados en la búsqueda de un nuevo contrato social: experiencias del Banco Intera- mericano de Desarrollo y la Red Tiempos. Rev Panam Salud Pública. 2005;17(5-6):438-43.

53. Souza M, Machado CV. Governança, intersetorialidade e participação social na política pública: o Conselho Nacional dos Direitos da Pessoa Idosa. Ciência \& Saúde Coletiva,. 2018;23:3189-200.

54. Goic A. El Sistema de Salud de Chile: una tarea pendiente. Rev Med Chil. 2015;143:774-86.

55. Pinedo LFV. Salud y calidad de vida en el adulto mayor. Rev Peru Med Exp Salud Publica. 2016;33(2):199-201.

56. Soto A, Burstein Z. Salud pública e investigación: la agenda pendiente. Rev Peru Med Exp Salud Publica. 2016;33(3):399-400.

57. Chiou-Tan FY, Rintala DH, Novy DM, Garza HM, High WM. J, Young ME. Psychometric properties of a Spanish-language version of the Community Integration Questionnaire (CIQ). Rehabil Psychol. 2005;47(2):144-64.

58. Van Brakel WH, Anderson AM, Mutatkar RK, Bakirtzief Z, Nicholls PG, Raju MS, et al. The Participation Scale: Measuring a key concept in public health. Disabil Rehabil. 2006;28(4):193-203.

59. Heffernan E, Coulson NS, Ferguson MA. Development of the Social Participation Restrictions Questionnaire (SPaRQ) through consultation with adults with hearing loss, researchers, and clinicians: a content evaluation study. Int J Audiol [Internet]. 2018;57(10):791-9. doi: https://doi.org/10. 1080/14992027.2018.1483585.

60. Levasseur M, Roy M, Michallet B, St-Hilaire F, Maltais D, Généreux M. Associations Between Resilience, Community Belonging, and Social Participation Among Community-Dwelling Older Adults : Results From the Eastern Townships Population Health Survey. Arch Phys Med Rehabil. 2017;98:2422-32.

61. Richard L, Gauvin L, Gosselin C, Laforest S. Staying connected: neighbourhood correlates of social participation among older adults living in an urban environment in Montréal, Quebec. Health Promot Int [Internet]. 2009 Mar;24(1):46-57. 